\title{
LAS RELACIONES CAMBIANTES ENTRE SISTEMA MEDIÁTICO Y SISTEMA DE PARTIDOS
}

\author{
En recuerdo de Fermín Bouza
}

\section{THE CHANGING RELATIONS BETWEEN THE MEDIA SYSTEM AND THE POLITICAL PARTY SYSTEM}

\author{
Juan Jesús González \\ UNED \\ jgonzalez@poli.uned.es \\ Palmira Chavero \\ FLACSO \\ pchavero@flacso.edu.ec
}

\section{Resumen}

Tras dos décadas de colaboración fraternal, Fermín Bouza tenía particular recuerdo del momento en que un grupo de investigadores de varias universidades madrileñas nos reunimos en torno a él para constituir el equipo que se llamó "Agenda y Voto". De manera que ningún tema resulta ahora tan oportuno para rendirle homenaje como el que pone en relación esas dos partes de la ecuación que durante mucho tiempo constituyó nuestra agenda de investigación, si bien la amplitud del periodo de referencia que vamos a utilizar en este artículo nos permite abrir el zoom y estudiar el contexto más amplio en que se desarrollan dicha relación. Así, por un lado, estudiaremos los cambios registrados en el sistema mediático como efecto combinado de la crisis de 2008 y del cambio de gobierno de 2011, en tanto que, por otro, los pondremos en relación con los cambios en el sistema de partidos tal como quedaron certificados en las elecciones generales de 2015. Utilizaremos los estudios postelectorales del CIS de 2008 y 2015 para poner de relieve la última fase de los cambios registrados en la evo- 
lución del pluralismo polarizado que preside el funcionamiento de nuestro sistema mediático, modificaciones que van de la mano en este caso de los cambios producidos con el advenimiento de la "nueva política".

Palabras clave: Sistema mediático, Sistema político, Polarización, Elecciones

\begin{abstract}
After two decades of fraternal collaboration, Fermín Bouza had a particular memory of the moment when a group of researchers from several universities in Madrid gathered around him to form the team called "Agenda y Voto". So that no issue is now as relevant to pay tribute as the one that relates those two parts of the equation that for a long time constituted our research agenda, although the breadth of the reference period that we are going to use in this article It allows opening the zoom and study the broader context in which this relationship develops. Thus, on the one hand, we will study the changes registered in the media system as a combined effect of the 2008 crisis and the 2011 change of government, while, on the other hand, we will put them in relation with the changes in the political party system as they were certified in the general elections of 2015. We will use the post-election studies of the CIS of 2008 and 2015 to highlight the last phase of the changes registered in the evolution of polarized pluralism that presides over the functioning of our media system, modifications that go from the hand in this case of the changes produced with the advent of the "new policy".
\end{abstract}

Keywords: Media system, Political system, Polarization, Elections

\title{
LA POLARIZACIÓN POLÍTICO-MEDIÁTICA
}

Una de las características más reseñables de la política española desde los años noventa ha sido su propensión a la polarización política, propensión que se explica, en primer lugar, por la influencia del sistema mediático, toda vez que nos encontramos con un sistema mediático de "pluralismo polarizado" (Hallin y Mancini, 2004). De hecho, la primera ola de polarización (al final de la primera etapa socialista) se produjo en un momento de máxima polarización mediática, a raíz de la liberalización del medio televisivo y la lucha consiguiente por la formación y el control de los nuevos conglomerados mediáticos. Como se recordará, en los primeros años noventa se configuraron, a grandes rasgos, los alineamientos político-mediáticos que sirvieron de caldo de cultivo a la primera ola de polarización promovida por Aznar (la alianza Polanco-Godó frente al llamado "sindicato del crimen"). En este caso, la confluencia político-mediática se apoyaba en un principio elemental de reparto de tareas: mientras el PP se 
dedicaba al acoso y derribo de Felipe González, los medios afines se dedicaban a explotar los escándalos de la etapa socialista, erosionando de esa manera la autoridad moral del gobierno socialista.

Más intrigante resulta la segunda fase de polarización, que se inicia en la segunda legislatura del PP, poco después de que este partido consiguiera la mayoría absoluta en las elecciones de 2000. Esta decisión estratégica de Aznar constituye el mejor ejemplo de que, con llamativa frecuencia, los partidos han preferido atender las demandas de sus propias bases, en vez de competir por el votante medio, como sería de esperar. Más concretamente, lo que este ejemplo sugiere es que los partidos se muestran receptivos a las demandas del votante medio mientras tratan de conseguir su voto, pero se radicalizan una vez que lo consiguen, momento a partir del cual traicionan los intereses de ese mismo votante medio mediante apuestas de alto riesgo electoral. ¿Cómo explicar esta opción estratégica por la polarización? Hay varias razones por las cuales la polarización resulta una opción racional de los partidos, entendiendo por tal una estrategia orientada a achicar el centro mediante incentivos para que sus potenciales habitantes lo abandonen, alineándose con alguno de los frentes en conflicto.

- La primera razón es que la polarización aumenta el sentimiento de inseguridad de los votantes menos ideológicos y más moderados, facilitando el cierre de filas en torno a dos bandos ideológicos que se convierten en trincheras (Corrales, 2005).

- La segunda razón es que la polarización induce respuestas simétricas del adversario político que generan una espiral de amenazas e incertidumbres, lo que refuerza el punto anterior (Ibíd.).

- La tercera es el achique del espacio ideológico de centro, como consecuencia del abandono del juego político y del consiguiente refugio en la abstención (Ansolabehere e Iyengar, 1995). La conversión de la política en un deporte agonístico que se agota en sí mismo, pero que no reporta mejora alguna en la convivencia y en la resolución de los problemas, induce sentimientos de rechazo, cuando no de cinismo ("todos son iguales"), desalentando la participación política de los moderados.

Ahora bien, a nadie se le escapa que estamos hablando de una empresa que desborda la capacidad de los partidos y que, de hecho, éstos no podrían asumir si no contasen con el concurso de los medios. Es igualmente obvio que la mencionada estrategia emprendida por José María Aznar hace ya más de una década no hubiera tenido recorrido de no ser por el acompañamiento mediático de que dispuso. El problema fue que, pese a los denodados esfuerzos del gobierno por contrarrestar la influencia del "imperio" Prisa mediante la creación de un holding mediático alternativo, Aznar terminó perdiendo esta batalla, como bien se pudo observar tras el 11-M de 2004 y el consiguiente vuelco electoral. 
A partir de ese momento, el panorama político-mediático se complicó extraordinariamente, como consecuencia de que a la competencia inter-bloques se sumó la competencia en el seno de cada uno de los bloques ideológicos. Así, en la legislatura 2004-2008, ABC y El Mundo compitieron por marcar la agenda del PP, en tanto que, en la siguiente (2008-2011), fueron Prisa y La Sexta quienes compitieron, a su vez, por influir sobre el gobierno socialista a favor de sus intereses respectivos. En este caso, el motivo de la división fue la promoción de un nuevo entramado mediático encabezado por La Sexta y el diario Público que, situado ideológicamente a la izquierda de los medios de Prisa, privó a ésta no sólo de su predominio en el campo audiovisual, sino también de su posición preferente como canal de comunicación entre el gobierno socialista y la audiencia. El activismo del gobierno socialista en el campo audiovisual alcanzó su clímax en el verano de 2009 con la aprobación por vía de urgencia de la TDT de pago, que permitió a Mediapro (productora de La Sexta) la explotación de los derechos del fútbol, con el consiguiente enfrentamiento con el grupo Prisa, que se había beneficiado de esa opción hasta entonces.

La emergencia del nuevo holding puso fin a la tradicional simbiosis entre Prisa y los gobiernos socialistas, de tal manera que, en un primer balance, esta dinámica de polarización político-mediática parecía diversificar el mapa audiovisual (al ampliarlo por la derecha y por la izquierda), al tiempo que complicaba los alineamientos en cada uno de los polos, pues a la tradicional competición entre izquierda y derecha se añade ahora la competición dentro de los bloques respectivos, con un mayor fraccionamiento ideológico y nuevos motivos de tensión y disputa en el seno de cada bloque.

Ahora bien, la irrupción de la crisis económica vino, por su parte, a revertir esta dinámica de mayor pluralismo mediante un proceso inverso de concentración corporativa. Dada la drástica contracción del mercado publicitario derivada de la crisis, el gobierno socialista decidió suavizar la regulación antimonopolio anterior y permitir la fusión de cadenas hasta el límite de tres operadores comerciales de ámbito nacional, lo que amenazaba con devolver el panorama televisivo al estadio anterior (en la época de Antena 3, Telecinco y Canal Plus). La incapacidad de los nuevos canales promovidos por el gobierno socialista (Cuatro, del grupo Prisa, que comenzó a operar en abierto en 2006, y La Sexta, que se incorporó al espectro televisivo poco después) para llegar a acuerdo alguno de fusión dejó la iniciativa en manos de los operadores con mayor control del mercado publicitario, de tal suerte que Telecinco absorbió a Cuatro y Antena 3 a La Sexta, restableciendo así una situación insólita en Europa, toda vez que tanto Telecinco como Antena 3 están supeditadas a capital italiano (Mediaset y Agostini). 
Todo lo cual planteaba numerosos e inquietantes interrogantes sobre el devenir del sistema mediático español ante el eventual regreso del PP al gobierno y el consiguiente retorno del ente público al viejo sistema de control gubernamental. Esta primera decisión del gobierno de Rajoy fue seguida poco después por una segunda decisión tendente a facilitar la fusión entre Antena 3 y La Sexta, pese a las recomendaciones en contra de la Comisión Nacional de la Competencia, que había advertido de los riesgos del nuevo duopolio (Mediaset y Antena 3), el cual pasaba a controlar casi el 90\% del mercado publicitario.

Por su parte, la radiotelevisión pública (RTVE) alcanzó durante el gobierno de Rodríguez Zapatero las cuotas de imparcialidad más elevadas de la democracia e incluso, por primera vez, asistimos a la elección de un director por consenso entre los dos principales partidos. De manera tal que se puede decir que en este período se dieron grandes pasos hacia la desgubernamentalización de la televisión pública, avances que desaparecieron con el retorno del PP.

Junto a esta involución del servicio público y la imparable concentración del medio televisivo privado, hay que tener en cuenta que la prensa de referencia ha estado sometida, por su parte, a una doble crisis derivada de: a) el cambio de modelo de negocio inducido por la irrupción de internet; y b) un proceso de financiarización, es decir, de progresiva dependencia del capital financiero tras la acumulación de deuda registrada en los años de la burbuja, todo lo cual no hacía sino radicalizar el tradicional conservadurismo de la prensa y arrojar dudas sobre la capacidad del sistema mediático para representar las distintas posiciones ideológicas.

Desde esta perspectiva, el caso del grupo Prisa constituye un ejemplo paradigmático de este proceso, después de amasar una deuda por importe de cinco mil millones de euros cuya explosión ha dado lugar a despidos masivos y al desplazamiento de la familia Polanco, antigua propietaria, en beneficio de fondos de capital riesgo y de la gran banca. Merece la pena que nos detengamos en este proceso, así como en las implicaciones del mismo en términos de línea editorial.

Espoleado por el éxito de su buque insignia (el diario El País), Prisa aprovechó su proximidad con los gobiernos de Felipe González para convertirse en un grupo multimedia amplio y diversificado, con presencia en casi todos los frentes de la industria cultural. En el año 2000 salió a Bolsa y entró en el selecto club de las principales empresas cotizadas (Ibex 35), poniendo en marcha diversas operaciones de apalancamiento financiero a fin de reforzar su posición en el sector de las plataformas digitales (Sogecable, Digital +), en un proceso típico de esos años de expansión corporativa basada en deuda (Almiron y Segovia, 2012). 
En 2010, con una deuda de cinco mil millones de euros y en pleno declive del negocio editorial, Juan Luis Cebrián, primer director de El País y posteriormente consejero delegado de Prisa, pone en marcha una operación de salvamento en negociación con grupos de capital riesgo que inyectan liquidez en el grupo. Como resultado de la negociación , Juan Luis Cebrián llegó a tener una remuneración anual de 13,6 millones de euros, equivalente al salario de varios cientos de redactores, en un momento en que el grupo registraba pérdidas por importe de 451 millones y estaba a punto de iniciar un ERE que afectaría a más de cien trabajadores (Almiron, 2013). Esta operación iría seguida de otra, ya con gobierno del PP, por la cual los principales acreedores de Prisa cambiaron deuda por participación en el accionariado, con el resultado de que la familia Polanco, que había tenido el control de Prisa, lo perdió en beneficio de la gran banca (Caixa, Santander...). Esta desventurada trayectoria de lo que había llegado a ser la principal industria cultural española y la que contaba con mayor proyección internacional constituye todo un ejemplo de lo que ha significado la entrada de capital financiero en el negocio periodístico, incluidas las inevitables acusaciones sobre el presunto giro conservador de su línea editorial.

Si tenemos en cuenta que el diario Público cerró su edición impresa poco después de la derrota socialista en las elecciones generales de 2011 (Informe Mongolia, 2013: Segunda parte), este giro ideológico de El País dejaba el campo de la izquierda huérfano de representación ideológica y de cobertura mediática, toda vez que el medio televisivo y la prensa de referencia pasaron a ser patrimonio casi exclusivo del campo conservador, de no ser por La Sexta, siempre y cuando su nuevo propietario (Atresmedia) estuviese dispuesto a cultivar el creciente nicho de audiencia que se abría a la izquierda de El País.

De acuerdo con el rediseño del mapa audiovisual auspiciado por Rodríguez Zapatero y elaborado por Miguel Barroso (primer Secretario de Estado de Comunicación del zapaterismo), el nuevo grupo creado en torno a La Sexta estaba llamado, como ya indicamos, a neutralizar y, en la medida de lo posible, a reemplazar la relación simbiótica que se había establecido entre el PSOE de Felipe González y el grupo Prisa (González, 2008). Pero, como es bien sabido, la crisis económica precipitó el fin del zapaterismo de tal forma que la sustitución de Rodríguez Zapatero se resolvió mediante un retorno al felipismo, encarnado en la figura de Pérez Rubalcaba, frente a las pretensiones sucesorias de Carmen Chacón, esposa del citado Barroso, que habían puesto su confianza en unas elecciones primarias que Rubalcaba se negó a convocar. A la hora de explicar esta negativa, hay pocas dudas de que una de las razones fue precisamente la dificultad del aparato socialista para desenvolverse en un entorno mediático cada vez más adverso, dada la creciente falta de sintonía entre Ferraz y La Sexta. 
Tras la fusión de La Sexta con Antena 3, el grupo Planeta (principal propietario) se convertía en la clave de bóveda del pluralismo polarizado como principio de articulación del espectro ideológico en la fluctuante democracia española. La novedad en este caso radica en que, a diferencia de la polarización de los años noventa y dos mil, que estaba protagonizada por distintos grupos multimedia que se erigían en representantes de cada uno de los bloques, los polos ideológicos se encuadran ahora dentro de un mismo grupo mediático, a resultas de la drástica concentración corporativa propiciada por la crisis económica. Una vez superados los temores iniciales de que la larga mano del gobierno influyese sobre Planeta con el fin de sacrificar uno de los polos en beneficio del otro, los directivos de La Sexta se sintieron libres de cualquier compromiso derivado de su procedencia socialista y exploraron a fondo el nuevo segmento de audiencia que se abría a la izquierda del PSOE como consecuencia de la crisis del bipartidismo.

\section{RELACIONES ENTRE LOS SISTEMAS MEDIÁTICO Y POLÍTICO}

El estudio postelectoral del CIS de enero-febrero de 2016 nos proporciona el estado actual de las relaciones entre el sistema mediático y el sistema de partidos al término del proceso que sucintamente acabamos de reseñar. Más concretamente, vamos a presentar información sobre la distribución de las audiencias de las principales cadenas de TV según el voto en las elecciones de diciembre de 2015. Esta información procede de la pregunta sobre cuál fue la cadena de televisión preferida para seguir la información política y electoral durante la campaña electoral. Para facilitar la correcta interpretación de los datos vamos a ordenar tanto los partidos como las cadenas de derecha a izquierda. Asimismo, vamos a presentar los datos de auto ubicación de los electorados de los partidos y de las audiencias de las televisiones con el fin de visualizar su posición en la escala ideológica.

Lo primero que llama la atención de la información contenida en la tabla adjunta es la notable correlación entre partidos y cadenas televisivas, lo que prueba el paralelismo político característico de los sistemas de pluralismo polarizado. Hay que tener en cuenta, por otra parte, que la distancia entre los partidos ha aumentado considerablemente ${ }^{1}$, lo que agudiza la polarización. Con estas premisas iniciales, hay dos cosas al menos que requieren comentario. La primera es que, como se recordará, la pasada legislatura empezó con la liquidación del Estatuto de RTVE de 2006 y la consiguiente gubernamentalización de este servicio público. Cabía esperar, por tanto, que el electorado más conservador y

\footnotetext{
1 Aunque Unidos Podemos aparece con posterioridad a las elecciones de 2015, hemos agrupado el voto de IU y Podemos, lo que convierte a esta categoría en segunda fuerza en número de votos, por delante del PSOE. Como resultado de esta agrupación, el votante medio de Podemos-IU se sitúa en el punto 3 de la escala ideológica, a cuatro puntos de distancia del votante del PP.
} 
envejecido del PP se refugiase en la 1 de TVE a la hora de informarse políticamente, lo que ha colocado la audiencia de esta cadena pública en el punto 5,5 de la escala ideológica (casi un punto a la derecha del votante medio). Ahora bien, lo más relevante de este panorama político-mediático es la aparición de una cadena, de parecida influencia a la que tiene la cadena pública ${ }^{2}$, cuya audiencia está situada casi un punto a la izquierda del votante medio, lo que la convierte en representante genuina del polo ideológico de la izquierda. En un régimen de pluralismo polarizado como el español, este dato es fundamental para entender las pretensiones de Podemos de convertirse en segunda fuerza electoral.

TABLA 1. Cadena preferida de información política, según voto 2015

\begin{tabular}{|c|c|c|c|c|c|c|c|c|}
\hline & \multicolumn{6}{|c|}{ Voto 2015} & \multirow[b]{2}{*}{ Total } \\
\hline & & PP & Cs & PSOE & Ps-IU & Otros & $\begin{array}{l}\text { Auto ubi- } \\
\text { cación }\end{array}$ & \\
\hline \multirow[t]{6}{*}{ Cadena } & TVE1 & $35,7 \%$ & $16,6 \%$ & $19,7 \%$ & $6,7 \%$ & $6,6 \%$ & 5,5 & $19,5 \%$ \\
\hline & A3 & $25,0 \%$ & $32,7 \%$ & $14,9 \%$ & $10,6 \%$ & $6,6 \%$ & 5,3 & $19,0 \%$ \\
\hline & Mediaset & $12,6 \%$ & $19,3 \%$ & $26,7 \%$ & $16,9 \%$ & $8,4 \%$ & 4,6 & $18,4 \%$ \\
\hline & La Sexta & $3,2 \%$ & $16,6 \%$ & $21,5 \%$ & $50,8 \%$ & $12,2 \%$ & 3,6 & $19,6 \%$ \\
\hline & Otras & $20,9 \%$ & $10,7 \%$ & $15,1 \%$ & $11,0 \%$ & $64,2 \%$ & 4,7 & $20,5 \%$ \\
\hline & $\mathrm{Ns} / \mathrm{Nc}$ & $2,5 \%$ & $4,1 \%$ & $2,1 \%$ & $3,9 \%$ & $2,1 \%$ & 4,7 & $3,0 \%$ \\
\hline \multicolumn{2}{|c|}{ Auto ubicación } & 7,0 & 5,5 & 3,7 & 3,0 & 3,7 & 4,7 & $100,0 \%$ \\
\hline
\end{tabular}

Como consecuencia de todo lo anterior, la probabilidad de votar a un partido está muy influida por la preferencia televisiva a la hora de informarse políticamente. Con el fin de precisar dicha influencia, la siguiente tabla presenta las probabilidades de votar a un partido según cuál sea la cadena preferida tomando como referencia la cadena o grupo de cadenas que están más próximas del votante medio. Pues bien, tomando como referencia los votantes que se informan preferentemente a través de Mediaset (Cuatro y Telecinco), la probabilidad de que un telespectador preferente de la 1 sea votante del PP se multiplica por 2,7, al tiempo que la probabilidad de que un telespectador preferente de La Sexta sea votante de Podemos o IU se multiplica por 3,7. Que una televisión gubernamental haya alcanzado una audiencia tan sesgada políticamente como la 1

\footnotetext{
2 Obsérvese que las cuatro cadenas que aparecen en la tabla tienen un volumen similar de menciones (en torno al 19\%), si bien hay que precisar que la categoría Mediaset se compone en realidad de dos cadenas (Cuatro y Telecinco).
} 
puede parecer un logro notable, pero no deja de palidecer ante el éxito sin precedentes de La Sexta.

TABLA 2. Probabilidades (odds ratios) de ser votante de un partido según la cadena preferida*

(Cat. Ref. Mediaset)

\begin{tabular}{|c|c|c|c|c|c|c|c|}
\hline & \multicolumn{6}{|c|}{ Voto 2015} \\
\hline & & PP & Cs & PSOE & Ps-IU & Otros & Total \\
\hline \multirow{6}{*}{$\begin{array}{l}\text { Ca- } \\
\text { dena }\end{array}$} & TVE1 & $2,7^{* * *}$ & & & & & $19,5 \%$ \\
\hline & A3 & $2,7^{* * *}$ & $2,7^{* * *}$ & $1,4^{* *}$ & & & $19,0 \%$ \\
\hline & $\begin{array}{l}\text { Me- } \\
\text { diaset }\end{array}$ & & & & & & $18,4 \%$ \\
\hline & $\begin{array}{l}\text { La } \\
\text { Sexta }\end{array}$ & & $1,9^{* * *}$ & $1,4^{* *}$ & $3,7^{* * *}$ & & $19,6 \%$ \\
\hline & Otras & & & & & $\begin{array}{r}5,5^{* *} \\
*\end{array}$ & $20,5 \%$ \\
\hline & $\mathrm{Ns} / \mathrm{Nc}$ & & & & & & $3,0 \%$ \\
\hline
\end{tabular}

${ }^{*}$ Controlando por ideología, edad y nivel de estudios.

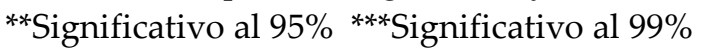

Con el fin de entender adecuadamente esta nueva polarización del sistema mediático, conviene volver la vista atrás y analizar la situación de 2008, por cuanto nos recuerda cuál era la situación anterior a la crisis económica (que está en el origen de los cambios en el sistema mediático) y a la crisis política (que está, a su vez, en el origen de los cambios en el sistema de partidos). Conviene comenzar por recordar, en este punto, que la televisión pública se regía entonces por el estatuto de 2006, que la consagraba como un auténtico servicio público, razón por la cual fue objeto de despolitización, a fin de que pudiera ser aceptada por igual entre los votantes de los diversos partidos. Los datos que vamos a presentar a continuación son una prueba concluyente de que esta operación fue realizada con éxito tanto en términos de audiencia (la Tve1 reunía el 28,9\% de las preferencias de los votantes) como de pluralidad electoral de esa misma audiencia (las probabilidades de que un televidente de TVE1 fuera votante socialista o votante popular eran muy parecidas, como veremos enseguida). Y conviene recordar, asimismo, que las nuevas cadenas que fueron licenciadas por el gobierno de Zapatero (Cuatro, concedida al grupo Prisa, y La Sexta, concedida a un grupo de nueva creación -Mediapro- auspiciado desde el gobierno) 
estaban todavía en una fase incipiente de implantación, por lo que las vamos a agrupar (entre las dos solo reunían el 7,4\% de las preferencias de los votantes).

Con estas premisas, la siguiente tabla nos informa del reparto de la audiencia según el voto en las elecciones de 2008. Como ya adelantamos, Tve1 se llevaba la mayor proporción de televidentes (28,9\%), seguida de Antena 3 (23,4\%) y Tele5 (16,2\%). Atendiendo a la relación entre audiencias y preferencias electorales, podemos observar una preferencia de los votantes del PP por A3 y de los del PSOE por Tve1. Ahora bien, si tomamos como referencia los votantes de Tele5 (que son los más próximos al votante medio) y controlamos por nivel de estudios y por ideología, vemos que las probabilidades de que los televidentes de la Tve1 voten por el PP o por el PSOE eran muy parecidas (ver tabla de probabilidades), lo que sugiere que la audiencia de Tve1 no estaba sesgada políticamente, al tiempo que prueba el éxito del estatuto de 2006 y la consiguiente despolitización de la RTVE.

TABLA 3. Cadena preferida de información política, según voto 2008

\begin{tabular}{|c|c|c|c|c|c|c|c|}
\hline & & PP & PSOE & Otros & Resto & $\begin{array}{c}\text { Auto } \\
\text { ubica- } \\
\text { ción }\end{array}$ & Total \\
\hline \multirow{6}{*}{$\begin{array}{l}\text { Ca- } \\
\text { dena }\end{array}$} & A3 & $36,5 \%$ & $14,9 \%$ & $14,6 \%$ & 21,9 & 5,5 & $23,4 \%$ \\
\hline & TVE1 & $28,2 \%$ & $35,8 \%$ & $16,8 \%$ & 25,6 & 4,8 & $28,9 \%$ \\
\hline & T5 & $14,0 \%$ & $19,8 \%$ & $13,4 \%$ & 15,6 & 4,6 & $16,2 \%$ \\
\hline & $\begin{array}{l}\text { Cua- } \\
\text { tro\&6 }\end{array}$ & $2,3 \%$ & $12,5 \%$ & $9,1 \%$ & 6,2 & 3,6 & $7,4 \%$ \\
\hline & Otras & $16,2 \%$ & $15,0 \%$ & $44,3 \%$ & 25,1 & 4,7 & $21,1 \%$ \\
\hline & $\mathrm{Ns} / \mathrm{Nc}$ & $2,8 \%$ & $1,9 \%$ & $1,8 \%$ & 5,6 & 5,1 & $3,0 \%$ \\
\hline \multicolumn{2}{|c|}{ Auto ubicación } & 6,6 & 3,6 & 3,9 & 4,7 & 4,8 & $\begin{array}{r}100,0 \\
\%\end{array}$ \\
\hline
\end{tabular}


TABLA 4.- Probabilidades (odds ratios) de ser votante de un partido según la cadena preferida*

(Cat. Ref. T5)

\begin{tabular}{|c|c|c|c|c|c|}
\hline & & $\mathrm{PP}$ & PSOE & Otros & Total \\
\hline \multirow[t]{6}{*}{ Cadena } & A3 & $2,4^{* * *}$ & & & $23,4 \%$ \\
\hline & TVE1 & $2,0^{* * *}$ & $2,2^{* * *}$ & & $28,9 \%$ \\
\hline & T5 & & & & $16,2 \%$ \\
\hline & $\begin{array}{l}\text { Cua- } \\
\text { tro\&6 }\end{array}$ & & $2,2^{* * *}$ & $1,6^{* *}$ & $7,4 \%$ \\
\hline & Otras & & & $2,8^{* * *}$ & $21,1 \%$ \\
\hline & $\mathrm{Ns} / \mathrm{Nc}$ & & & & $3,0 \%$ \\
\hline
\end{tabular}

\section{CONCLUSIONES}

Pese a que la reforma de RTVE de 2006 iba en la buena dirección, por cuanto RTVE dejaba de ser un instrumento del gobierno de turno y se erigía en un verdadero servicio público, la crisis económica y financiera, por un lado, y el retorno del PP al gobierno, por otro, se confabularon para liquidar uno de los aspectos más reivindicables de la herencia de Rodríguez Zapatero. Primero, la radicalización de las demandas sociales como consecuencia de la crisis y, más tarde, el sometimiento de Tve1 a la disciplina gubernamental alimentaron una dinámica incesante de polarización ideológica que llevó el pluralismo polarizado característico del sistema mediático español a niveles sin precedentes. De nuevo nos encontramos ante un sistema caracterizado por la existencia de dos polos ideológicos antagónicos con escasa pluralidad interna en cada uno de ellos, facilitando de esta manera una fuerte correspondencia entre el sistema mediático y el sistema de partidos, en virtud de la cual la incomunicación y el bloqueo que se observan entre la clase política amenaza con trasladarse a la esfera pública.

Con estos antecedentes, caben dos posibilidades para RTVE: o bien la vuelta a una fórmula parecida a la empleada en la etapa de Rodríguez Zapatero o bien la exploración de fórmulas innovadoras que permitan la despolitización del ente público por otras vías. En cualquiera de los casos, este sería el primer paso ineludible para desmontar el modelo de pluralismo polarizado tal como está configurado en el caso español. Lo demás queda de momento a merced de las fuerzas del mercado y de la capacidad de los medios privados para conciliar sus intereses corporativos con los de sus respectivas audiencias. 


\section{REFERENCIAS}

Almiron, Núria (2013): “Board compensation in the media industry: Global elite networks and financialization", The Political Economy of Communication, Vol. 1, No 2.

Almiron, Núria y Ana I. Segovia (2012): “Financialization, Economic Crisis, and Corporate Strategies in Top Media Companies: The Case of Grupo Prisa", International Journal of Communication 6, 2894-2917.

Ansolabehere, Stephen y Shanto Iyengar (1995): Going Negative. How Political Advertisements Shrink \& Polarize the Electorate, New York: The Free Press.

Corrales, Javier (2005): "In Search of a Theory of Polarization: Lessons from Venezuela, 1999-2005", Revista Europea de Estudios Latinoamericanos y del Caribe $79,105-118$.

Hallin, Daniel y Paolo Mancini (2004): Comparing Media Systems. Three Models of Media and Politics, Cambridge: Cambridge University Press.

Informe Mongolia (2013): Papel mojado. La crisis de la prensa y el fracaso de los periódicos en España, Barcelona: Debate.

JUAN JESÚS GONZÁLEZ RODRÍGUEZ. Catedrático de Sociología en la UNED. Ha realizado estancias en prestigiosas universidades como la Universidad de Wisconsin-Madison y la Universidad Politécnica de California. Es autor de numerosos libros y publicaciones de temática social y política, entre ellos “Tres décadas de cambio social en España" (en colaboración con Miguel Requena) y “Las razones del voto en la España democrática. 1977-2008” (junto con Fermín Bouza). En los últimos tiempos ha investigado en el campo de la Comunicación Política: "Agenda de los medios y voto", "Agenda mediática y agenda ciudadana", proyectos ambos patrocinados por el CIS. Es, además, profesor del Máster de Estudios Avanzados en Comunicación Política, que se imparte en la UCM.Catedrático de Opinión Pública en la Universidad Complutense de Madrid. Investigador y escritor, especializado en el estudio de los medios de comunicación, la realidad política y el comportamiento electoral.

PALMIRA CHAVERO. Profesora e investigadora del programa de Estudios Internacionales y Comunicación en FLACSO (Ecuador). Doctora en Ciencias de la Comunicación y Sociología por la Universidad Complutense de Madrid (España); Licenciada en Periodismo por la misma universidad. Investigadora del equipo de investigación Agenda y Voto, del Grupo de Investigación en Go- 
bierno, Administración y Políticas Públicas (Gigapp); de proyectos de investigación internacionales (Journalistic Role Performance around the world; Worlds of Journalism Study; Culturas Periodísticas de Ecuador). Ha trabajado en la Universidad Complutense de Madrid (España), el Instituto Universitario de Investigación Ortega y Gasset y ha sido coordinadora del Laboratorio de Comunicación y Derechos (Instituto de Altos Estudios Nacionales, Ecuador). En 2011 recibió el "I Premio Jóvenes Investigadores Joan Prats", concedido por Gigapp y el Instituto Universitario de Investigación Ortega y Gasset. Coautora de una decena de libros, de artículos en revistas especializadas y de numerosas ponencias en congresos internacionales. Ha trabajado como periodista durante más de diez años; en 2008 recibió el "Premio Periodismo 8 de marzo".

Recibido: 09/09/2017

Aceptado: 01/10/2017 